\title{
The effects of application of an ancient type of acupuncture needle on body temperature, immune function and the autonomic nerve system
} \author{
Toru Abo ${ }^{1}$ \\ ${ }^{1}$ Department of Immunology, Niigata University School of Medicine, Niigata, Japan; \\ "Corresponding Author: immunol2@med.niigata-u.ac.jp \\ ${ }^{2}$ Urahigashi Veterinary Clinic, Osaka, Japan \\ ${ }^{3}$ School of Health Sciences, Faculty of Medicine, Niigata University, Niigata, Japan \\ ${ }^{4}$ Aoyama Madarame Clinic, Tokyo, Japan \\ ${ }^{5}$ Fukuda Clinic, Niigata, Japan
}

Mayumi Watanabe ${ }^{1^{*}}$, Osamu Takano ${ }^{2 *}$, Chikako Tomiyama ${ }^{3}$, Hiroaki Matsumoto ${ }^{1}$, Nobuatsu Urahigashi ${ }^{2}$, Eisuke Kainuma ${ }^{1}$, Takeo Madarame ${ }^{4}$, Minoru Fukuda ${ }^{5}$,

Received 27 August 2012; revised 25 September 2012; accepted 3 October 2012

\section{ABSTRACT}

The di-zhen (DZ) is an ancient type of acupuncture needle with a history dating back more than 2000 years. Unlike modern acupuncture needles, the DZ is not inserted subcutaneously, and is safely and commonly used at the bedside. The mechanisms underlying its effects are not known. In this study, we measured sublingual and cutaneous body temperature, pulse rate, oxygen pressure $\left(\mathrm{PO}_{2}\right)$, oxygen saturation $\left(\mathrm{SO}_{2}\right)$ and carbon dioxide pressure $\left(\mathrm{PCO}_{2}\right)$ before and after DZ application in 25 healthy male volunteers. Serum levels of catecholamines (adrenaline, noradrenaline and dopamine) and white blood cells (WBCs; ratio and number) were traced for one week. Soon after DZ application, pulse rate, body temperature, $\mathrm{PO}_{2}$ and $\mathrm{SO}_{2}$ all decreased. The serum levels of adrenaline and noradrenaline increased, indicating sympathetic dominance, and the number of granulocytes was elevated. One week after DZ application, the number of lymphocyte increased. We therefore suggest that $D Z$ affects body temperature, pulse rate, catecholamine secretion and immune function by inducing transient sympathetic dominance via actions on the autonomic nervous system. These effects are similar to the effects observed with modern needles, which are inserted subcutaneously. Therefore, we consider DZ treatment to be advantageous and safe in modern clinical practice, especially in post-

*These authors contributed equally to this work. surgical and terminal care, as it avoids the issues with infection and tissue damage sometimes seen with modern acupuncture needles.

Keywords: Di-Zhen (DZ); Acupuncture; Body Temperature; Autonomic Nervous System (ANS); White Blood Cells (WBC); Immune System

\section{INTRODUCTION}

Findings from the Tyrolean Iceman, who had tattoos at acupuncture points along the Bladder Meridian, have been suggested to possibly reflect the ancient origin of acupuncture [1]. Acupuncture is still widely practiced in East Asia, but the detailed mechanisms underlying its effects are poorly characterized. One of the earliest forms of acupuncture needle was the "bian-shi" (a piece of edged stone) in the Neolithic age, and this developed into the metal "Nine kinds of needles" as described in the "Huangdi Neijing" (Yellow Emperor's classic of internal medicine) of the Western Han dynasty about 2 BC [2]. The "di-zhen" (DZ), a pressing-type needle, represents one of these nine kinds of metal needles, and it is often used at the bedside. Unlike modern acupuncture needles, the DZ is not inserted subcutaneously. In this study, we tested the effects of DZ application on body temperature, immune function and the autonomic nerve system. We chose to study DZ for several reasons. First, the treatment has been used for thousands of years and is therefore time-tested and standardized. Second, the DZ is a non-invasive needle, which minimizes the possibility of infection or tissue damage. Third, the great methodological variability seen during the long history of acupuncture makes it difficult to evaluate the mechanisms of 
acupuncture therapy. The DZ represents the origin of modern acupuncture, and its application is simple and common. To quantify physiological response to DZ application, we analyzed body temperature, catecholamine levels, blood gases, hemoglobin $(\mathrm{Hb})$ and the ratio and the absolute number of white blood cells (WBCs). The evaluation of these data enables us to shed new light on the fundamental mechanisms of modern acupuncture.

\section{MATERIALS AND METHODS}

\subsection{Subjects}

Twenty-five healthy male volunteers, 24 to 60 years of age, participated in this study. Written informed consent was obtained from all subjects and the study was approved by the institutional review board of Niigata University.

\subsection{DZ Application}

The DZ needles were made of stainless steel and had a column-like body and a flat head (Soken Medical Co. Ltd., Toshima, Japan). An autoclaved DZ was softly pressed vertically onto the body surface for 5 minutes (diameter $5.0 \mathrm{~mm}, 37.3 \mathrm{~g} / \mathrm{mm}^{2}$ ) (Figure 1(a)), at approximately 120 points from the head to the feet on both sides of the spine (Figure 1(b)). The DZ application points also corresponded to the Bladder Meridian as defined by the World Health Organization [3]. The treatment was applied by nationally licensed experienced Japanese acupuncturists.

\subsection{Selection of Subjects for Assessment}

For measurement of body temperature and pulse rate before and after DZ treatment, the subjects were divided randomly into two age-matched groups. Half of the subjects $(\mathrm{n}=13 ; 37.4 \pm 12.6$ years) received $\mathrm{DZ}$ application and the matched controls $(\mathrm{n}=12 ; 37.8 \pm 13.7$ years $)$ received no treatment. For measurements other than body temperature and pulse rate, six randomly selected subjects $(34.5 \pm 10.9$ years $)$ received DZ treatment and were compared to six age-matched controls $(33.1 \pm 14.3$ years $)$.

\subsection{Measurement of Body Temperature}

Core temperature (sublingual) was measured with a clinical mercury thermometer (FAVOR, Nihon Keiryoki Kogyo Co. Ltd., Chiyoda, Japan) before and after DZ application. The cutaneous body temperature was measured using a contactless digital thermometer (Microlife, IR 1DA1, microlife, Switzerland) before and after DZ at five locations: Yintang (on the forehead, mid-way between the medial ends of the eyebrows), LI4 (Hegu; on the dorsum of the right and left hands, radial to the mid-point of the second metacarpal bone), LR3 (Taichong; on the
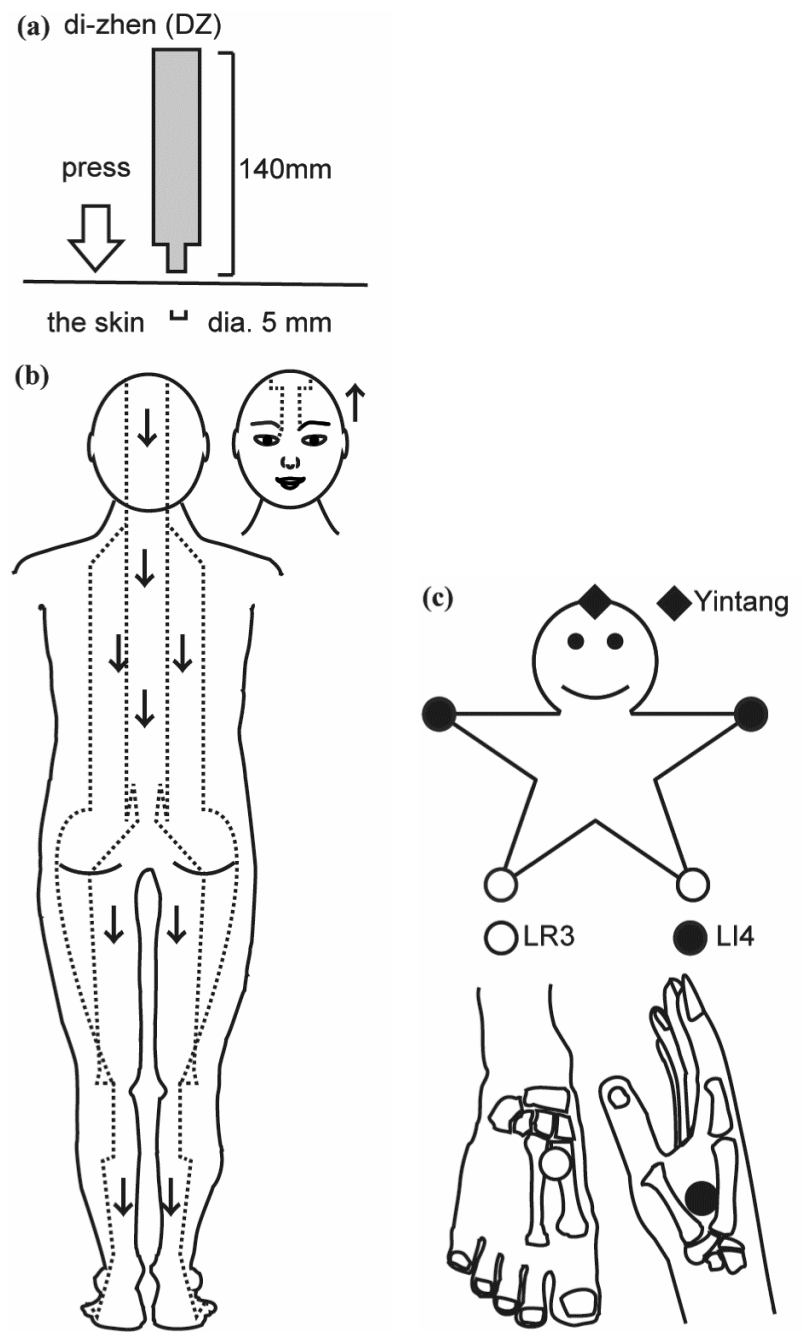

Figure 1. (a) The DZ needle, made of stainless steel and with a column-like body and a flat head, was used vertically to exert soft pressure on the skin of the subjects; (b) Over 120 applied points were stimulated from the head to the feet on both sides of the spine. This needle placement corresponded to the Bladder Meridian as defined by the World Health Organization; (c) The cutaneous body temperature was measured at the indicated five points: Yintang (forehead), LI4 (Hegu; on the dorsum of right and left hand), LR3 (Taichong; on the dorsum of the right and left feet).

dorsum of the right and left feet, between the first and second metatarsal bones, in the depression distal to the junction of the bases of the two bones, over the dorsalis pedis artery) (Figure 1(c)).

\subsection{Assessment of Other Parameters}

Fresh venous blood was obtained before and after the $\mathrm{DZ}$ application for the measurement of $\mathrm{PO}_{2}, \mathrm{SO}_{2}, \mathrm{PCO}_{2}$ and hemoglobin, using i-STAT $300 \mathrm{~F}$ (i-STAT Corporation, East Windsor, NJ, USA) as previously described [4-7]. WBCs were measured using a hemocytometer and the May-Grünwald Giemsa stain method. Concentrations 
of catecholamines (adrenaline, noradrenaline and dopamine) were measured by high-performance liquid chromatography (SRL, Inc., Tokyo, Japan). Additionally WBCs and catecholamines were examined at one week after DZ application.

\subsection{Statistical Analysis}

The statistical significance of differences between values was tested using paired $t$-tests or repeated-measures two-factor ANOVA and a Bonferroni/Dunn post-hoc test. $P$ values of less than 0.05 were considered to be statisticcally significant.

\section{RESULTS}

\subsection{Pulse Rate and Sublingual Body Temperature}

Pulse rate and sublingual body temperature were measured before and soon after DZ application. The pulse rate showed a prominent decrease after DZ application (from $71.4 \pm 4.7$ to $66.2 \pm 5.4 / \mathrm{min}, P<0.05$ ), but the body temperature did not change significantly $(36.8 \pm 0.3$ versus $\left.36.6^{\circ} \mathrm{C} \pm 0.4^{\circ} \mathrm{C}, P>0.05\right)$. No changes were seen in the control group (Figure 2(a)).

\subsection{Blood Gases and Hemoglobin}

Because a drop in pulse rate might affect blood circulation and thereby cause a decrease in the oxygen content of venous blood, we measured oxygen and carbon dioxide pressure $\left(\mathrm{PO}_{2}\right.$ and $\left.\mathrm{PCO}_{2}\right)$ and oxygen saturation $\left(\mathrm{SO}_{2}\right)$. We found that $\mathrm{PO}_{2}$ and $\mathrm{sO}_{2}$ both decreased significantly after DZ application $(28.9 \pm 10.9$ to $19.4 \pm$ $10.5 \mathrm{mmHg}, P<0.05$; and $46.8 \% \pm 9.4 \%$ to $24.8 \% \pm 9.1 \%$, $P<0.05$, respectively). These changes tended to recover within 60 minutes after DZ application; however, this apparent return to baseline was not statistically significant owing to high inter-subject variability (data not shown). Neither $\mathrm{PCO}_{2}$ nor $\mathrm{Hb}$ showed any prominent changes. In the control group, no changes were observed in any of the parameters (Figure 2(b)).

\subsection{Cutaneous Body Temperature}

It has been reported that rapid changes in core temperature is associated with changes in cutaneous blood flow mediated by the autonomic nervous system (ANS) [8]. Although it was not statistically significant DZ application induced a tendency of a rapid decrease in core temperature (Figure 2(a)). Therefore, we measured cutaneous body temperature over time at five points (Figure 1(c)). Soon after DZ stimulation, the temperature of the hands and feet appeared to transiently decrease (average: hands, $\triangle 0.7^{\circ} \mathrm{C} \pm 0.6^{\circ} \mathrm{C}$; feet, $\triangle 1.9^{\circ} \mathrm{C} \pm 1.5^{\circ} \mathrm{C}$ ) then began to recover after 20 minutes. However, these

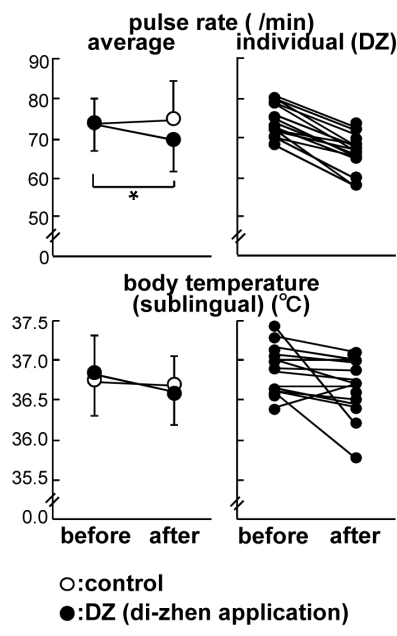

(a)

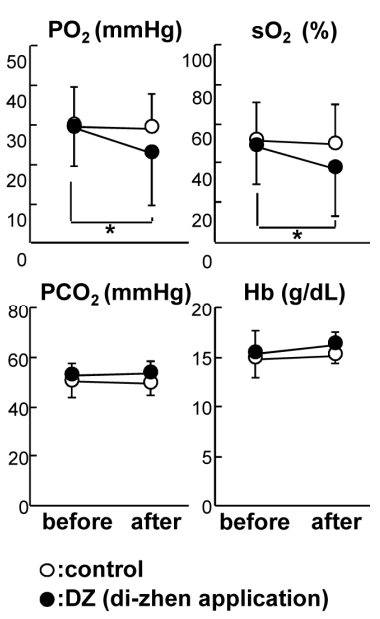

(b)
Figure 2. (a) The pulse rate and body temperature (sublingual) before and after DZ application. The average values and individual data of the DZ application group are shown. ${ }^{*} P<0.05$. Both the pulse rate and body temperature decreased after DZ application in almost all subjects. No such changes were seen in the control group; (b) $\mathrm{PO}_{2}$ and $\mathrm{SO}_{2}$ significantly decreased after DZ application. Neither $\mathrm{PCO}_{2}$ nor $\mathrm{Hb}$ showed any changes. No prominent change was observed in any parameter in the control group.

changes were not statistically significant $(P>0.05)$ (Figure 3).

\subsection{Levels of Adrenaline, Noradrenaline and Dopamine}

The levels of catecholamines are known to reflect the status of the ANS [9-11]; thus, these were measured before and after the DZ application. We found that the serum levels of adrenaline and noradrenaline were significantly elevated after DZ treatment (from $33 \pm 7$ to $39 \pm$ $10 \mathrm{pg} / \mathrm{mL}, P<0.05$; and from $216 \pm 69$ to $306 \pm 92$ $\mathrm{pg} / \mathrm{mL}, P<0.05$; respectively). Dopamine did not change significantly (Figure 4(a)). One week after DZ application, there was no statistically significant difference in catecholamine levels compared to baseline (data not shown).

\subsection{The Ratio and Number of WBCs}

The above finding of an elevation in adrenaline and noradrenaline indicates dominance of sympathetic nervous activity. Mori et al. reported that electric acupuncture affected ANS status and had an immunomodulatory effect [9]. To investigate the effect of DZ application on immune function and ANS status the number and ratio of WBCs were studied.

After DZ application the ratio of granulocytes appeared to increase $(66 \% \pm 6 \%$ to $61 \% \pm 9 \%, P>0.05)$ while that of lymphocyte tend to decrease though they were not 


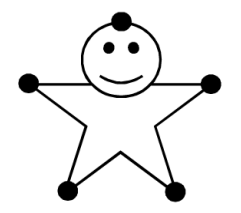

cutaneous body temperature five surface points $\left({ }^{\circ} \mathrm{C}\right)$
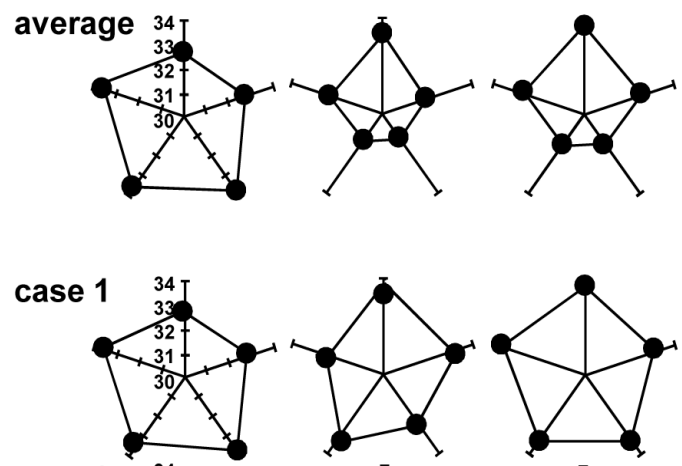

case 2
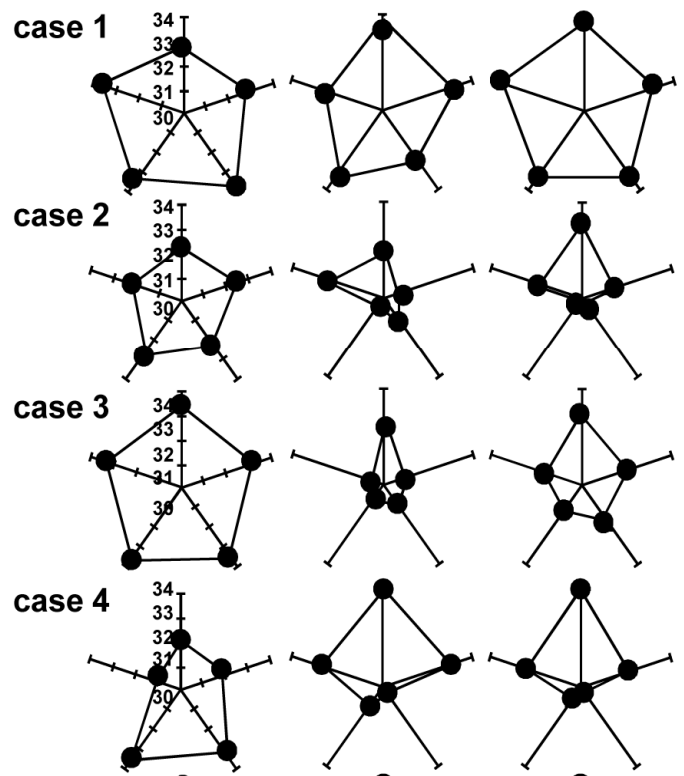

case 5
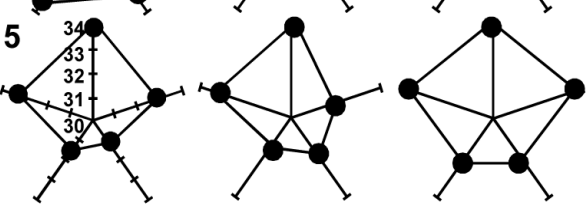

case 6

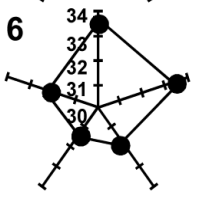

before

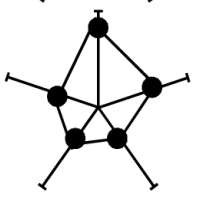

after

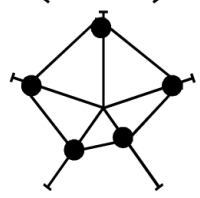

$20 \mathrm{~min}$
Figure 3. The temporal variation of the cutaneous body temperature at five points (Figure 1(c)) are illustrated, before, immediately after and 20 minutes after DZ application. Soon after stimulation, both hands and both feet showed a tendency towards a transient decrease followed by recovery within 20 to 40 minutes.

statistically significant ( $33 \% \pm 6 \%$ to $37 \% \pm 8 \%, P>0.05$ ) (Figure 4(b)). The absolute numbers of granulocytes and lymphocytes were determined by calculation. The number of lymphocytes showed a step-like elevation and there was a statistically significant difference between levels at baseline versus at one week after treatment $(2159 \pm 90$ to $2485 \pm 51 / \mu \mathrm{L})(P<0.05)$ (Figure 4(b)). catecholamine (pg/mL)
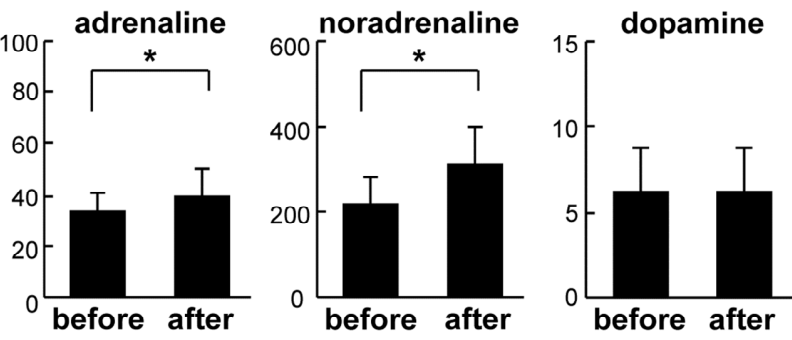

(a)
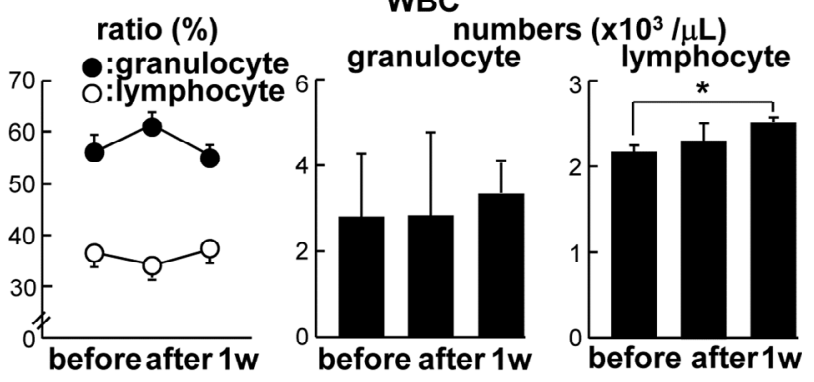

(b)

Figure 4. (a) Changes in the levels of catecholamines (adrenaline, noradrenaline and dopamine) after DZ application. The serum levels of adrenaline and noradrenaline were significantly elevated while those of dopamine did not show any prominent change. ${ }^{*} P<0.05$; (b) The ratio and the absolute number of WBCs (granulocytes and lymphocytes) were investigated before, immediately after and one week after DZ application. The ratio of granulocytes tended to increase while that of lymphocytes tended to decrease. The number of lymphocytes showed a step-like elevation between baseline and one week after treatment. ${ }^{*} P<0.05$.

\section{DISCUSSION}

\subsection{DZ Application Induced Sympathetic Dominance}

In a series of previous studies, we have found body temperature to be an important parameter in understanding metabolic conditions induced by changes in ANS activity and immune status $[6,10,11]$. The sublingual and cutaneous temperature decreased after DZ application though they were not statistically significant (Figures 2(a) and 3), while the levels of adrenaline and noradrenaline increased (Figure 4(a)). Because adrenaline and noradrenaline are known to be elevated under conditions of sympathetic dominance [11,12], it is likely that the observed elevation in catecholamines reflect an increase in sympathetic activity induced by DZ application. Adrenaline $\alpha$-receptors are involved in mediating decreases in body temperature [11]. It has been reported that adrenaline $\beta$-receptors mediate the constriction of heart or vascular smooth muscle and decreases in pulse rate caused by acupuncture via ANS modulation $[9,13]$. Our results are consistent with these previous reports. 


\subsection{Transience of the Sympathetic Dominance Induced by DZ Application}

A continuous state of high sympathetic activity induces adaptive responses to maintain homeostasis $[10,11$, 14,15]. However, the responses caused by DZ application were transient, with recovery of the cutaneous hypothermia seen within 20 minutes (Figure 3). In this pilot study, we confirmed that the decreased pulse rate and sublingual hypothermia also showed recovery within 20 to 40 minutes, and that the levels of catecholamines recovered the following week. While the great inter-individual variability prevented firm conclusions, the changes in all subjects showed the same trend toward recovery (data not shown). This supports the hypothesis that the changes in sympathetic status induced by DZ application were transient.

\subsection{DZ Application Affected the Ratio and Number of WBC via ANS Modulation}

DZ application induced a rapid change in the WBC profile. The number of lymphocytes did not change on the day of the application but showed a step-like elevation after one week (Figure 4(b)). Thus it is undeniable that DZ application affected the ratio and number of WBCs via ANS modulation, as previously reported [16].

\subsection{Comparison between DZ Application and Other Skin Stimulation}

We recently investigated the effect of skin rubdown with a dry towel (SRDT), which stimulated almost the same part of the skin [4]. Interestingly, DZ application and SRDT induced precisely the opposite effects on pulse rate and sublingual body temperature, even though they are both considered to affect the immune system via ANS modulation. One reason may be found in the difference of the stimuli applied with the two methods. DZ application stimulated a very narrow point, while SRDT mildly stimulated a wider area of the skin. The effect of DZ application was much stronger than that of SRDT. In fact, the changes in adrenaline level and noradrenaline level after DZ application were 1.2 times and 1.3 times greater than those of SRDT [4]. This might indicate that these two treatments applied different doses of stimulation and that the dose of the DZ application was stronger than that of SRDT. Another reason may be differences in the location of the stimulated tissue. It is possible that stimulation with stronger pressures may stimulate deeper components of the tissue. Last, a possible reason might be the compatibility of the ancient type of acupuncture needle with the stimulated acupuncture points along the Bladder Meridian. If this historically reported compatibility is significant in the effect of DZ compared to other treat- ments, it should be possible to observe effects that are specific for DZ application. Further research is required to support this hypothesis.

\section{CONCLUSION}

In this study we have shown that the use of the 2000year-old acupuncture needle DZ exerted effects on body temperature, pulse rate, catecholamine secretion and immune system function via ANS modulation. Moreover, although the DZ is not inserted subcutaneously like modern needles, the observed effects of DZ treatment were similar to effects of application of modern needles, which are inserted under the skin. Therefore, despite its ancient origins, DZ is useful in modern clinical practice, especially in the case of postsurgical care, infection and terminal stage care. As infection and tissue damage caused by subcutaneous insertion of acupuncture needles can be a problem $[17,18]$, DZ application may provide a better solution that avoids these issues.

\section{ACKNOWLEDGEMENTS}

The authors thank Ms. Hiromi Ishiwatari (Soken Medical Co. Ltd.), Mr. Taiki Hashimoto and Ms. Kaori Yamamoto (Yushima-Shimizuzaka Clinic), Dr. Ryoko Fukuda (Fukuda Clinic), Dr. Takahiro Kobayashi and Urahigashi Veterinary Clinic for arrangement of the clinical research.

\section{REFERENCES}

[1] Dorfer, L., Moser, M., Spindler, K., Bahr, F., Egarter-Vigl, E. and Dohr, G. (1998) 5200-year-old acupuncture in central Europe? Science, 282, 242-243. doi:10.1126/science.282.5387.239f

[2] Ma, K. (1992) The roots and development of Chinese acupuncture: From prehistory to early 20th century. $\mathrm{Acu}$ puncture in Medicine, 10, 92-99. doi:10.1136/aim.10.Suppl.92

[3] World Health Organization Western Pacific Region (2008) WHO standard acupuncture point locations in the western pacific region. World Health Organization Western $\mathrm{Pa}$ cific Regional Office, Manila.

[4] Watanabe, M., Takano, O., Tomiyama, C., Matsumoto, H., Kobayashi, T., Madarame, T. and Abo, T. (2012) Skin rubdown with a dry towel, "kanpu-masatsu", is an aerobic exercise. Biomedical Research, 33, 243-248.

[5] Ohishi, T., Nukuzuma, C., Seki, A., Watanabe, M., Tomiyama-Miyaji, C., Kainuma, E., Inoue, M., Kuwano, Y. and Abo, T. (2009) Alkalization of blood pH is responsible for survival of cancer patients by mild hyperthermia. Biomedical Research, 30, 95-100. doi:10.2220/biomedres.30.95

[6] Tomiyama-Miyaji, C., Watanabe, M., Ohishi, T., Kanda, Y., Kainuma, E., Bakir, H.Y., Shen, J., Ren, H., Inoue, M., Tajima, K., Bai, X. and Abo, T. (2007) Modulation of the endocrine and immune systems by well-controlled hy- 
perthermia equipment. Biomedical Research, 28, 119-125. doi:10.2220/biomedres.28.119

[7] Watanabe, M., Miyajima, K., Matsui, I., Tomiyama-Miyaji, C., Kainuma, E., Inoue, M., Matsumoto, H., Kuwano, Y. and Abo, T. (2010) Internal environment in cancer patients and proposal that carcinogenesis is adaptive response of glycolysis to overcome adverse internal conditions. Health, 2, 781-788. doi: $10.4236 /$ health.2010.27118

[8] Baker, M.A., Cronin, M.J. and Mountjoy, D.G. (1976) Variability of skin temperature in the waking monkey. American Journal of Physiology, 230, 449-455.

[9] Mori, H., Nishijo, K., Kawamura, H. and Abo, T. (2002) Unique immunomodulation by electro-acupuncture in humans possibly via stimulation of the autonomic nervous system. Neuroscience Letters, 320, 21-24. doi:10.1016/S0304-3940(02)00012-5

[10] Kainuma, E., Watanabe, M., Tomiyama-Miyaji, C., Inoue, M., Kuwano, Y., Ren, H. and Abo, T. (2009) Association of glucocorticoid with stress-induced modulation of body temperature, blood glucose and innate immunity. Psychoneuroendocrinology, 34, 1459-1468. doi:10.1016/i.psyneuen.2009.04.021

[11] Watanabe, M., Tomiyama-Miyaji, C., Kainuma, E., Inoue, M., Kuwano, Y., Ren, H., Shen, J. and Abo, T. (2008) Role of alpha-adrenergic stimulus in stress-induced modulation of body temperature, blood glucose and innate immunity. Immunology Letters, 115, 43-49. doi:10.1016/j.imlet.2007.09.010

[12] Suzuki, S., Toyabe, S., Moroda, T., Tada, T., Tsukahara, A., Iiai, T., Minagawa, M., Maruyama, S., Hatakeyama,
K., Endoh, K. and Abo, T. (1997) Circadian rhythm of leucocytes and lymphocytes subsets and its possible correlation with the function of the autonomic nervous system. Clinical \& Experimental Immunology, 110, 500-508. doi:10.1046/j.1365-2249.1997.4411460.x

[13] Tanaka, T.H., Leisman, G. and Nishijo, K. (1997) The physiological responses induced by superficial acupuncture: A comparative study of acupuncture stimulation during exhalation phase and continuous stimulation. International Journal of Neuroscience, 90, 45-58.

[14] McEwen, B.S. (2000) Allostasis and allostatic load: Implications for neuropsychopharmacology. Neuropsychopharmacology, 22, 108-124. doi:10.1016/S0893-133X(99)00129-3

[15] McEwen, B.S. (2004) Protection and damage from acute and chronic stress, allostasis and allostatic overload and relevance to the pathophysiology of psychiatric disorders. Annals of the New York Academy of Sciences, 1032, 1-7. doi:10.1196/annals.1314.001

[16] Abo, T. and Kawamura, T. (2002) Immunomodulation by the autonomic nervous system: Therapeutic approach for cancer, collagen diseases, and inflammatory bowel diseases. Therapeutic Apheresis, 6, 348-357. doi:10.1046/j.1526-0968.2002.00452.x

[17] Walsh, B. (2001) Control of infection in acupuncture. Acupuncture in Medicine, 19, 109-111. doi:10.1136/aim.19.2.109

[18] Woo, P.C., Lin, A.W., Lau, S.K. and Yuen, K.Y. (2010) Acupuncture transmitted infections. British Medical Journal, 340, 1147-1152. doi:10.1136/bmj.c1268 